\title{
Flow Cytometric Evaluation of Human Neutrophil Apoptosis During Nitric Oxide Generation In Vitro: The Role of Exogenous Antioxidants
}

\author{
Zofia Sulowska, ${ }^{1}$ Ewa Majewska, ${ }^{2}$ Magdalena Klink, ${ }^{1}$ Malgorzata Banasik, ${ }^{3}$ and Henryk Tchórzewski ${ }^{3}$ \\ ${ }^{1}$ Center for Medical Biology, Polish Academy of Sciences, 93-232 Lodz, Poland \\ ${ }^{2}$ Department of Laboratory Diagnostic and Clinical Biochemistry, Medical University, 90-153 Lodz, Poland \\ ${ }^{3}$ Department of Clinical Immunology, Polish Mother's Memorial Institute, 93-338 Lodz, Poland
}

Received 9 December 2004; accepted 21 December 2004

\begin{abstract}
Among numerous inflammatory mediators a nitric oxide molecule is supposed to be important in the modulation of neutrophil survival in vivo and in vitro. The effect of exogenous supply of NO donors such as SNP, SIN-1, and GEA-3162 on the course of human neutrophil apoptosis and the role of extracellular antioxidants in this process was investigated. Isolated from peripheral blood, neutrophils were cultured in the presence or absence of NO donor compounds and antioxidants for 8, 12, and 20 hours. Apoptosis of neutrophils was determined in vitro by flow cytometric analysis of cellular DNA content and Annexin V protein binding to the cell surface. Exposure of human neutrophils to GEA-3162 and SIN-1 significantly accelerates and enhances their apoptosis in vitro in a time-dependent fashion. In the presence of SNP, intensification of apoptosis has not been revealed until 12 hours after the culture. The inhibition of GEA-3162- and SIN-1-mediated neutrophil apoptosis by superoxide dismutase (SOD) but not by catalase (CAT) was observed. Our results show that SOD and CAT can protect neutrophils against NO-donors-induced apoptosis and suggest that the interaction of $\mathrm{NO}$ and oxygen metabolites signals may determine the destructive or protective role of NO donor compounds during apoptotic neutrophil death.
\end{abstract}

\section{INTRODUCTION}

Normal human neutrophils spontaneously undergo apoptosis in vitro and in vivo after their migration from the vascular compartment to the tissue. However, their death by apoptosis can be triggered and accelerated or delayed by a number of different extracellular or intracellular stimuli including inflammatory mediators such as cytokines, reactive oxygen species (ROS), and nitric oxide (NO) $[1,2,3,4]$. On the other hand activated neutrophils are a known source of ROS which are required for killing invading microorganisms $[5,6]$. The apoptotic neutrophils are recognized and removed by macrophages without liberating their toxic intracellular contents from them $[7,8,9,10]$. Control of the neutrophil apoptosis process plays an important role in the resolution of acute and chronic inflammation.

NO has been recognized as an important mediator of various neutrophils activities including their chemotaxis, ROS generation and degranulation [11, 12, 13]. NO donors are pharmacologically active compounds that liberate NO in vivo and in vitro. They differ in

Correspondence and reprint requests to Zofia Sulowska, Center for Medical Biology, Polish Academy of Sciences, 93-232 Lodz, Poland; zsulowska@cbm.pan.pl; suzo@wp.pl their chemical structures, stability, and biological activity $[14,15]$. Compounds such as sodium nitroprusside (SNP) [16], 3-morpholinosydnonimine (SIN-1), a molsidomine metabolite, and 3-aryl-substituted oxatriazole5-imine derivative, GEA-3162 [17], besides their effect on vascular smooth muscle cells, affect circulating white blood cells by inhibiting adhesion and aggregation of neutrophils $[18,19]$. The amount and duration of NO liberated from the respective NO donors determine their pharmacological properties and usefulness as drugs in the therapy of patients with acute lung injury or coronary heart disease [16]. Numerous experiments have demonstrated the regulatory role of $\mathrm{NO}$ in apoptosis but the mechanisms by which this molecule exerts its protective or destructive role during apoptotic cell death still require clarification.

The aim of this study was to investigate the effect of exogenous supply of SNP, SIN-1, and GEA-3162 on the course of apoptosis in normal human neutrophils and the role of extracellular antioxidants in this process.

\section{MATERIALS AND METHODS}

\section{Reagents}

1, 2, 3, 4-oxatriazolium-5-amino-3-(3,4-dichlorophenyl)-chloride (GEA-3162) was from Alexis, Switzerland. 
3-morpholinosydnonimine hydrochloride (SIN-1), sodium nitroprusside (SNP), trypan blue, propidium iodide (PI), superoxide dismutase (SOD), catalase (CAT), fetal calf serum (FCS), $100 \mathrm{U} / \mathrm{mL}$ of penicillin and $100 \mu \mathrm{g} / \mathrm{mL}$ of streptomycin, ethylenediaminetetraacetate (EDTA), ribonuclease A (RNase A) were obtained from Sigma (Sigma Chemical Co, St Louis, Mo). Phosphate-buffered saline (PBS), RPMI 1640 were purchased from Biomed (Lublin, Poland). Polymorphprep was obtained from Nycomed (Oslo, Norway). Annexin V/FITC Kit was purchased from Bender MedSystems Diagnostics $\mathrm{GmbH}$ (Vienna, Austria). Twenty-four-well flat-bottom plates were from NUNC Brand Products (Denmark). MayGrünwald-Giemsa dyes were purchased from AQUAMED (Lodz, Poland).

\section{Cell preparation}

Neutrophils were isolated from heparinized peripheral blood collected from healthy donors using a onestep density-gradient centrifugation on Polymorphprep at $450 \times g$ at room temperature for 30 minutes. The residual erythrocytes were removed from the cell population by hypotonic lysis. The neutrophils were washed twice and resuspended in PBS [20]. The cell suspensions were stored in PBS at a concentration of $1 \times 10^{6} / \mathrm{mL}$ at $4^{\circ} \mathrm{C}$ and used within 2 hours. Cell purity (more than 98\%) and viability (more than $98 \%$ ) were determined by MayGrünwald-Giemsa staining of cytocentrifuged samples and by Trypan blue exclusion, respectively.

Informed consent was obtained from all donors. The Local Ethical Committee accepted this study.

\section{Culture conditions of normal human neutrophils}

Neutrophils were maintained in RPMI 1640 supplemented with 10\% FCS and antibiotics: $100 \mathrm{U}$ of penicillin and $100 \mu \mathrm{g} / \mathrm{mL}$ of streptomycin. Two milliliters of cell suspension $\left(1 \times 10^{6} / \mathrm{mL}\right)$ were placed in 24 -well plates, and duplicate conditions were cultured in humidified atmosphere at $37^{\circ} \mathrm{C}$ with $5 \% \mathrm{CO}_{2}$ for varying periods of time as indicated. Neutrophil suspensions were incubated in the absence (control cell samples) or presence of $10 \mu \mathrm{M}$ of GEA-3162, $1 \mathrm{mM}$ of SNP, and $1 \mathrm{mM}$ of SIN- 1 in the presence or not of exogenous CAT $(1200 \mathrm{U} / \mathrm{mL})$ and SOD $(300 \mathrm{U} / \mathrm{mL})$. The cells were cultured for 8,12 , and 20 hours and next washed once in PBS. Aliquots were removed and used in assays of neutrophil apoptosis.

Flow cytometric detection of PMN apoptosis by measurement of Annexin V (ANX-FITC) binding to cell surface and analysis of cellular DNA content was performed as described earlier [21].

\section{Measurement of fluorescein isothiocyanate-labeled Annexin V (ANX-FITC) binding to cell surface}

The percentage of apoptotic neutrophils was determined using the binding of ANX-FITC protein to cell surface according to the manufacturer's specification. Briefly, specific binding of ANX-FITC was performed by incuba- tion of cell suspension $\left(1 \times 10^{5}\right.$ cells $)$ in $100 \mu \mathrm{L}$ of binding buffer with $2 \mu \mathrm{L}$ of ANX-FITC and $10 \mu \mathrm{L}$ of PI (final concentration $1 \mu \mathrm{g}$ ) for 25 minutes in the dark at room temperature. After incubation, cells were centrifuged and analyzed using a FACScan flow cytometer (Becton Dickinson, Heidelberg, Germany) equipped with Cell Quest software for cells acquisition and data analysis.

\section{Analysis of DNA content by PI staining and flow cytometry}

Briefly, the PMNs $\left(10^{6} / 200 \mu \mathrm{L}\right)$ after washing with PBS supplemented with $0.5 \mathrm{mM}$ EDTA were resuspended in ice-cold $70 \%$ ethanol and stored at $-20^{\circ} \mathrm{C}$ for one to two days. Then, neutrophils were treated with RNase A $(10 \mu \mathrm{g} / \mathrm{mL})$ and stained with $20 \mu \mathrm{g} / \mathrm{mL}$ of PI. Cells were held in the dark at room temperature for 15 minutes and then stored at $4^{\circ} \mathrm{C}$ until flow cytometric analysis. The fluorescence of individual nuclei was measured using Cell Quest software for cell acquisition and data analysis. The percentage of cells with apoptotic nuclei (located as a hypodiploid DNA peak in the DNA fluorescence histogram) was calculated.

\section{Statistical analysis}

The results are expressed as means \pm standard deviations (SD) of at least 5 independent experiments carried out with neutrophils from different individuals. Evaluation of statistical significance was performed by Wilcoxon's signed rank test. The value $P \leq .05$ was considered significant.

\section{RESULTS}

The nitrite generation from structurally distinct NO donors was measured using Griess reagent as described earlier [22] and on the basis of these data the doses of NO donors were calculated and used in all subsequent experiments. The effect of NO donors on ROS production by neutrophils was also described earlier [22]. The percentage of apoptotic neutrophils in a culture treated with $\mathrm{NO}$ donors evaluated by flow cytometry is shown in Figure 1. The data are presented as the mean of percentage of the sum of cells binding Annexin V (ANX-FITC+PI- and ANX-FITC+PI+). Exposure of human neutrophils to exogenous NO donors such as GEA-3162 and SIN-1 significantly accelerates and enhances their apoptosis in vitro in a time-dependent fashion. In the presence of SNP the attenuation of neutrophil apoptosis was noted after 8 hours of culture, however, small but significant intensification of apoptosis was observed after 12 hours of neutrophil culture. The similar course of neutrophil apoptosis in the presence of NO donors was confirmed by evaluation of cellular DNA staining as a second marker of apoptosis [23] (Figure 2). The comparative analysis of cell apoptosis after 8 and 12 hours of cultures evaluated by cellular DNA content and ANX-FITC binding and PI staining in the same sample of neutrophil cultures is presented 



FIGURE 1. Effect of NO donors on neutrophil apoptosis in vitro measured by ANX-FITC and PI binding. Neutrophils $\left(1 \times 10^{6}\right.$ cells) were incubated in the absence (control cell samples) (open bars) or presence of $10 \mu \mathrm{M}$ of GEA-3162, $1 \mathrm{mM}$ of SNP, and $1 \mathrm{mM}$ of SIN- 1 at $37^{\circ} \mathrm{C}$ (closed bars) for 8,12 , and 20 hours and apoptosis was determined as described in "materials and methods." Data are given as means and SD percentages of the sum of cells binding ANX-FITC+PI- and ANX-FITC+PI+ for 9 separate experiments. Statistical significance: neutrophils versus neutrophils with nitric oxide donors $(*$ denotes that $P \leq .005, * *$ denotes that $P \leq .05$ ).

in Table 1. This analysis allows to show the presence of neutrophils in different stages of apoptosis [24, 25]. In the presence of GEA-3162 and SIN-1 but not SNP in the culture medium, the cells expressing phosphatidylserine (PS) at the plasma membrane and binding Annexin V (an early stage of apoptosis) are dominated in the first 8 hours of cultures. These changes at the plasma membrane in SNP-treated neutrophils were not revealed until 12 hours after the culture. To eliminate the release of superoxide


FIGURE 2. Effect of nitric oxide donors on neutrophil apoptosis in vitro measured by DNA content. Neutrophils $\left(1 \times 10^{6}\right.$ cells $)$ were incubated at $37^{\circ} \mathrm{C}$ with indicated stimuli (closed bars) or in the medium (open bars) for 8, 12, and 20 hours and apoptosis was determined as described in "materials and methods." Data are presented as the means and SD percentages of cells with apoptotic nuclei. Values are statistically significant: neutrophils versus neutrophils with nitric oxide donors $(*$ denotes that $P=.003, * *$ denotes that $P=.002$ ).

radicals from the cells environment to extracellular media and prevent the formation of ROS and other derivatives during NO donors decomposition, the antioxidants such as SOD and CAT were added to neutrophils culture and neutrophil survival in vitro was investigated. As shown in Table 2, the presence of SOD $(300 \mathrm{U} / \mathrm{mL})$ in the culture media markedly inhibited the spontaneous neutrophil apoptosis. The addition of SOD to the culture media significantly reduced GEA-3162- and SIN-1-induced neutrophil apoptosis after 8 hours of culture, however, this effect of SOD was not observed after 20 hours of culture. In contrast, presence of SOD did not modify the SNP-mediated neutrophil apoptosis at the same time of culture. As presented in Table 3 the addition of CAT to the culture of neutrophils treated with SNP caused a significant decrease in the cells binding ANX-FITC and PI after 8 hours and 20 hours of culture. 
TABLE 1. Comparative analysis of apoptosis evaluated by ANX-FITC binding and cellular DNA content in the same sample of neutrophil culture. Neutrophils $\left(1 \times 10^{6}\right.$ cells $)$ were incubated with the indicated stimuli for 8 and 12 hours and apoptosis was determined as described in "materials and methods." Results are given as means and SD percentages of apoptotic cells for 6 separate experiments. Statistical significance: neutrophils versus neutrophils with NO donors ( $*$ denotes that $P \leq .05 ; * *$ denotes that $P=.027$ ).

\begin{tabular}{c|cc|cc}
\hline & \multicolumn{2}{|c|}{ \% of ANX-FITC+PI- and ANX-FITC+PI+ cells } & \multicolumn{2}{c}{ \% of cells with apoptotic nuclei } \\
\hline Agents & \multicolumn{2}{|c|}{ Time of neutrophils culture } & \multicolumn{2}{c}{ Time of neutrophils culture } \\
\hline & $8 \mathrm{~h}$ & $12 \mathrm{~h}$ & $8 \mathrm{~h}$ & $12 \mathrm{~h}$ \\
\hline Medium & $28 \pm 8$ & $57 \pm 11$ & $23 \pm 7$ & $49 \pm 17$ \\
GEA-3162 & $51 \pm 1^{* *}$ & $73 \pm 2^{*}$ & $24 \pm 4$ & $61 \pm 21^{* *}$ \\
Medium & $23 \pm 8$ & $48 \pm 8$ & $15 \pm 6$ & $17 \pm 6$ \\
SIN-1 & $62 \pm 15^{* *}$ & $78 \pm 5^{* *}$ & $24 \pm 7^{*}$ & $29 \pm 13^{*}$ \\
SNP & $23 \pm 6$ & $55 \pm 12^{*}$ & $15 \pm 1$ & $27 \pm 8^{* *}$ \\
\hline
\end{tabular}

TABLE 2. Effect of exogenous superoxide dismutase (SOD) on apoptosis of neutrophils nontreated and treated by NO donors in vitro measured by ANX-FITC and PI binding. Neutrophils $\left(1 \times 10^{6}\right.$ cells $)$ were incubated at $37^{\circ} \mathrm{C}$ with the indicated stimuli in the presence of SOD $(300 \mathrm{U} / \mathrm{mL})$ or not for 8 and 20 hours and apoptosis was determined as described in "materials and methods." Results are given as means and SD percentages of the sum of cells binding ANX-FITC+PI- and ANX-FITC+PI+ for 6 separate experiments. Statistical significance: $* *$ denotes neutrophils with SOD versus neutrophils with GEA-3162, SIN-1 $(P \leq .05)$; $*$ denotes neutrophils with GEA-3162+SOD and with SIN-1+SOD versus neutrophils with GEA-3162 and SIN-1, respectively $(P \leq .05)$. nd means no data.

\begin{tabular}{c|cc}
\hline & \multicolumn{2}{|c}{ \% of ANX-FITC+PI- and ANX-FITC+PI+ cells } \\
\hline Agents & \multicolumn{2}{c}{ Time of culture } \\
\hline & $8 \mathrm{~h}$ & $42 \pm 17$ \\
\hline SOD & $15 \pm 3$ & $90 \pm 3^{* *}$ \\
GEA-3162 & $51 \pm 9^{* *}$ & $84 \pm 3$ \\
GEA-3162+SOD & $38 \pm 13^{*}$ & $88 \pm 6^{* *}$ \\
SIN-1 & $82 \pm 13^{* *}$ & $84 \pm 4$ \\
SIN-1+SOD & $65 \pm 10^{*}$ & nd \\
SNP & $23 \pm 6$ & nd \\
SNP+SOD & $26 \pm 7$ & \\
\hline
\end{tabular}

\section{DISCUSSION}

To study the apoptotic process in time we used two flow cytometric methods: PS translocation by Annexin V binding and DNA fragmentation by PI staining. In Figures 1 and 2 the effect of NO donors on neutrophils apoptosis regarding the different time of cultures (measured by Annexin V and PI binding or DNA fragmentation) is shown. This set of experiments was carried out on neutrophils derived from different blood donors. In Table 1 , by comparison of these two cytometric methods, we present the analysis of the kinetics of different features of apoptosis of neutrophils treated by NO donors. Because PS translocation precedes endonuclease activation and DNA fragmentation we compare the detection of different stages of neutrophils apoptosis in the same sample of cell culture. PS translocation is followed rapidly (within 1-2 hours) by nucleosomal DNA fragmentation and for this reason we limited time points of detection to 8 and 12 hours.

All the three compounds studied liberate NO during its decomposition in an aqueous solution in vitro in a dose- and time-dependent manner. GEA-3162, SNP, and SIN-1 differ in their time and amount of NO release, when used in the same experiment conditions. We have shown earlier that SNP released NO in the cell-free system; however, the presence of neutrophils enhanced its generation [22]. They also differ in a number of other metabolites, which arise during decomposition of a given compound [26], some of them through spontaneous release of NO simultaneously form a superoxide anion $\left(\mathrm{O}_{2}{ }^{-}\right)$[14]. It was well documented that some $\mathrm{NO}$ donors in an aqueous solution form peroxynitrite $\left(\mathrm{ONOO}^{-}\right)$spontaneously as well as from endogenous superoxide anion and liberated NO. SIN-1 is the NO and superoxide anion donor and is also regarded as $\mathrm{ONOO}^{-}$donor. Formation of $\mathrm{ONOO}^{-}$ in a solution of GEA-3162 has been also shown but its amount was negligible as compared to SIN-1 [16, 17, 27].

We show here that the exposure of human neutrophils to exogenous NO donors accelerates and enhances their apoptosis in vitro in a time-dependent fashion. Our results are in accordance with other reports that NO is able to trigger and accelerate the apoptosis in neutrophils and in this way can modulate the course of inflammation $[28,29,30,31,32]$. The analysis of the course of neutrophil apoptosis confirms the hypotheses of Taylor et al that different compounds depending on the mechanisms of NO liberation exert differential effects on apoptotic events $[33,34]$. 
TABLE 3. Effect of exogenous catalase (CAT) on apoptosis of neutrophils nontreated and treated by NO donors in vitro, measured by ANX-FITC and PI binding. Neutrophils $\left(1 \times 10^{6}\right.$ cells $)$ were incubated with the indicated stimuli in the presence of CAT $(1200 \mathrm{U} / \mathrm{mL})$ or not for 8 and 20 hours and apoptosis was determined as described in "materials and methods." Results are given as means and SD percentages of the sum of cells binding ANX-FITC+PI- and ANX-FITC+PI+ for 6 separate experiments. Statistical significance: $* *$ denotes neutrophils with CAT versus neutrophils with NO donors, $*$ denotes neutrophils with SNP versus neutrophils with $\mathrm{SNP}+\mathrm{CAT}(P \leq .05)$.

\begin{tabular}{c|cc}
\hline & \multicolumn{2}{|c}{$\%$ of ANX-FITC+PI- and ANX-FITC+PI+ cells } \\
\hline Agents & \multicolumn{2}{c}{ Time of culture } \\
\hline & $19 \pm 5 \mathrm{~h}$ & $33 \pm 10^{* *}$ \\
CAT & $73 \pm 7^{* *}$ & $79 \pm 5^{* *}$ \\
GEA-3162 & $71 \pm 10$ & $71 \pm 13$ \\
GEA-3162+CAT & $67 \pm 10^{* *}$ & $76 \pm 12^{* *}$ \\
SIN-1 & $65 \pm 8$ & $70 \pm 18$ \\
SIN-1+CAT & $44 \pm 16$ & $57 \pm 9$ \\
SNP & $26 \pm 7^{*}$ & $40 \pm 15^{*}$ \\
\hline
\end{tabular}

The data presented in Tables 2 and 3 are derived from separate set experiments which were conducted during a few months and carried out on neutrophils derived from different healthy subjects. The discrepancy between the data given in Tables 2 and 3 is most likely due to neutrophil donors and their ability to undergo apoptosis as well as the time (season) of blood sample collecting. Ward et al [30] also noticed some variability in the levels of induction of apoptosis between experiments which, according to them, was mainly due to donor variations. The influence of season on immune cells function is considered and discussed. The susceptibility and reactivity of circulating blood cells varies at different stages of their circadian and seasonal (circannual) rhythms. Seasonal variations in the function of blood cells were observed and it was documented by others that cells collected in autumn were more active than those sampled in spring $[35,36,37]$. We also observed the differences in neutrophils response to the stimuli dependently on the season of blood collecting and individual variations in the rate of response of neutrophils to exogenous stimuli were also noticed by us.

Many external stimuli that lead to neutrophil apoptosis also induce these cells to the generation of reactive species. ROS are known intracellular mediators of neutrophil apoptosis $[3,38,39,40]$. We found earlier that neither SNP nor SIN-1 and GEA-3162 alone induce $\mathrm{O}_{2}^{-}$ generation in human neutrophils [22]. However, these results cannot exclude the possibility that ROS released from neutrophils to the surrounding milieu during their long-term culturing in vitro may promote their death by apoptosis. Exogenously added SOD and CAT are cell membrane impermeable scavengers and are thus suitable as inhibitors of oxidative metabolites release to the extracellular media. Others and we have demonstrated that SOD and CAT have a protective role against the induction of neutrophil apoptosis [4, 39, 40, 41]. We examined the involvement of these antioxidants in neutrophil apoptosis induced by NO donor compounds. In our system SOD attenuated the apoptosis of nontreated and NOdonor-treated neutrophils. The inhibition of GEA-3162and SIN-1-mediated neutrophil apoptosis by SOD suggests that superoxide anion generated during SIN-1 and GEA-3162 decomposition contributes to the apoptotic effect of NO donors. However, it cannot be excluded that peroxynitrite which is also formed during the donor decomposition participates in cell death. In contrast, neutrophil apoptosis in the presence of GEA-3162 and SIN-1 was not modified by the addition of CAT. In turn, the inhibition of neutrophil apoptosis by CAT in the presence of SNP suggests that the apoptotic effect was dependent on the release of $\mathrm{H}_{2} \mathrm{O}_{2}$ from cells rather than on SNP action. The effect of exogenously supplied antioxidants on the course of apoptosis process in NO-donor-treated neutrophils demonstrates the presence of ROS that can appear during NO compound decomposition or/and longterm neutrophil culturing and their involvement in apoptotic cells death. Our results show that SOD and CAT can protect neutrophils against $\mathrm{NO}$-donor-induced apoptosis and suggest that the interaction of NO and ROS signals may determine the destructive or protective role of $\mathrm{NO}$ donor compounds during apoptotic neutrophil death. It is not clear whether the protection was mediated directly by the scavenging of superoxide anion or indirectly by regulating some other signal transduction pathway.

\section{REFERENCES}

[1] Colotta F, Re F, Polentarutti N, Sozzani S, Mantovani A. Modulation of granulocyte survival and programmed cell death by cytokines and bacterial products. Blood. 1992;80(8):2012-2020.

[2] Lee A, Whyte MK, Haslett C. Inhibition of apoptosis and prolongation of neutrophil functional longevity by inflammatory mediators. J Leukoc Biol. 1993;54(4):283-288. 
[3] Buttke TM, Sandstrom PA. Oxidative stress as a mediator of apoptosis. Immunol Today. 1994;15(1):710.

[4] Watson RWG. Redox regulation of neutrophil apoptosis. Antioxid Redox Signal. 2002;4(1):97-104.

[5] Miller RA, Britigan BE. The formation and biologic significance of phagocyte-derived oxidants. J Investig Med. 1995;43(1):39-49.

[6] Babior BM. The respiratory burst of phagocytes. $J$ Clin Invest. 1984;73(3):599-601.

[7] Savill JS, Wyllie AH, Henson JE, Walport MJ, Henson PM, Haslett C. Macrophage phagocytosis of aging neutrophils in inflammation. Programmed cell death in the neutrophil leads to its recognition by macrophages. J Clin Invest. 1989;83(3):865-875.

[8] Hart SP, Haslett C, Dransfield I. Recognition of apoptotic cells by phagocytes. Experientia. 1996;52(10-11):950-956.

[9] Savill J. Apoptosis in resolution of inflammation. J Leukoc Biol. 1997;61(4):375-380.

[10] Fadok VA, Chimini G. The phagocytosis of apoptotic cells. Semin Immunol. 2001;13(6):365-372.

[11] Armstrong R. The physiological role and pharmacological potential of nitric oxide in neutrophil activation. Int Immunopharmacol. 2001;1(8):1501-1512.

[12] Bogdan C, Röllinghoff M, Diefenbach A. Reactive oxygen and reactive nitrogen intermediates in innate and specific immunity. Curr Opin Immunol. 2000;12(1):64-76.

[13] Morikawa $M$, Inoue $M$, Tokumaru S, Kogo $H$. Enhancing and inhibitory effects of nitric oxide on superoxide anion generation in human polymorphonuclear leukocytes. $\mathrm{Br} \mathrm{J}$ Pharmacol. 1995;115(7):1302-1306.

[14] Yamamoto T, Bing RJ. Nitric oxide donors. Proc Soc Exp Biol Med. 2000;225(3):200-206.

[15] Moilanen E, Vapaatalo H. Nitric oxide in inflammation and immune response. Ann Med. 1995;27(3):359-367.

[16] Megson IL. Nitric oxide donor drugs. Drugs of the Future. 2000;25:701-715.

[17] Kankaanranta H, Rydell E, Petersson AS, et al. Nitric oxide-donating properties of mesoionic 3-aryl substituted oxatriazole-5-imine derivatives. $\mathrm{Br}$ J Pharmacol. 1996;117(3):401-406.

[18] Hogg N, Darley-Usmar VM, Wilson MT, Moncada S. Production of hydroxyl radicals from the simultaneous generation of superoxide and nitric oxide. Biochem J. 1992;281 (pt 2):419-424.

[19] Moilanen E, Vuorinen P, Kankaanranta H, MetsäKetelä T, Vapaatalo H. Inhibition by nitric oxidedonors of human polymorphonuclear leucocyte functions. Br J Pharmacol. 1993;109(3):852-858.

[20] Klink M, Cedzynski M, St Swierzko A, Tchórzewski $\mathrm{H}$, Sulowska Z. Involvement of nitric oxide donor compounds in the bactericidal activity of human neutrophils in vitro. J Med Microbiol. 2003;52(pt 4):303-308.
[21] Sulowska Z, Majewska E, Krawczyk K, Klink M, Tchórzewski H. Influence of opioid peptides on human neutrophil apoptosis and activation in vitro. Mediators Inflamm. 2002;11(4):245-250.

[22] Klink M, Tchórzewski H, Sulowska Z. Oxidative and adhesive responses of human neutrophils to nitrovasodilators in vitro: the role of protein kinases. Mediators Inflamm. 2003;12(6):345-353.

[23] Nicoletti I, Migliorati G, Pagliacci MC, Grignani F, Riccardi C. A rapid and simple method for measuring thymocyte apoptosis by propidium iodide staining and flow cytometry. J Immunol Methods. 1991;139(2):271-279.

[24] Darzynkiewicz Z, Bruno S, Del Bino G, et al. Features of apoptotic cells measured by flow cytometry. Cytometry. 1992;13(8):795-808.

[25] Vermes I, Haanen C, Steffens-Nakken H, Reutelingsperger C. A novel assay for apoptosis. Flow cytometric detection of phosphatidylserine expression on early apoptotic cells using fluorescein labelled Annexin V. J Immunol Methods. 1995;184(1):39-51.

[26] Feelisch M. The biochemical pathways of nitric oxide formation from nitrovasodilators: appropriate choice of exogeneous NO donors and aspects of preparation and handling of aqueous NO solution. J Cardiovasc Pharmacol. 1991;17(suppl 3):S25-S33.

[27] Holm P, Kankaanranta H, Metsä-Ketelä T, Moilanen E. Radical releasing properties of nitric oxide donors GEA 3162, SIN-1 and Snitroso-N-acetylpenicillamine. Eur J Pharmacol. 1998;346(1):97-102.

[28] Blaylock MG, Cuthbertson BH, Galley HF, Ferguson NR, Webster NR. The effect of nitric oxide and peroxynitrite on apoptosis in human polymorphonuclear leukocytes. Free Radic Biol Med. 1998;25(6):748-752.

[29] Brüne B, von Knethen A, Sandau KB. Nitric oxide and its role in apoptosis. Eur J Pharmacol. 1998;351(3):261-272.

[30] Ward C, Wong TH, Murray J, et al. Induction of human neutrophil apoptosis by nitric oxide donors: evidence for a caspase-dependent, cyclicGMP-independent, mechanism. Biochem Pharmacol. 2000;59(3):305-314.

[31] Melino G, Catani MV, Corazzari M, Guerrieri P, Bernassola F. Nitric oxide can inhibit apoptosis or switch it into necrosis. Cell Mol Life Sci. 2000;57(4):612-622.

[32] Kim PKM, Zamora R, Petrosko P, Billiar TR. The regulatory role of nitric oxide in apoptosis. Int Immunopharmacol. 2001;1(8):1421-1441.

[33] Taylor EL, Megson IL, Haslett C, Rossi AG. Dissociation of DNA fragmentation from other hallmarks of apoptosis in nitric oxide-treated neutrophils: differences between individual nitric oxide donor drugs. Biochem Biophys Res Commun. 2001;289(5):12291236. 
[34] Taylor EL, Megson IL, Haslett C, Rossi AG. Nitric oxide: a key regulator of myeloid inflammatory cell apoptosis. Cell Death Differ. 2003;10(4):418-430.

[35] Linden M, Larson M, Prellner T, Brattsand R, Laitinen LA. Seasonal variation in the function of blood monocytes obtained from healthy nonsmokers, asymptomatic smokers, and smokers with chronic bronchitis. Chronobiol Int. 1994;11(4):266272.

[36] Maes M, Stevens W, Scharpe S, et al. Seasonal variation in peripheral blood leukocyte subsets and in serum interleukin-6, and soluble interleukin-2 and 6 receptor concentrations in normal volunteers. Experientia. 1994;50(9):821-829.

[37] Nelson RJ, Demas GE, Klein SL, Kriegsfeld LJ. The influence of season, photoperiod, and pineal melatonin on immune function. J Pineal Res. 1995;19(4):149-165.

[38] Kasahara Y, Iwai K, Yachie A, et al. Involvement of reactive oxygen intermediates in spontaneous and CD95 (Fas/APO-1)-mediated apoptosis of neutrophils. Blood. 1997;89(5):1748-1753.

[39] Oishi K, Machida K. Inhibition of neutrophil apoptosis by antioxidants in culture medium. Scand J Immunol. 1997:45(1):21-27.

[40] Rollet-Labelle E, Grange MJ, Elbim C, Marquetty C, Gougerot-Pocidalo MA, Pasquier C. Hydroxyl radical as a potential intracellular mediator of polymorphonuclear neutrophil apoptosis. Free Radic Biol Med. 1998;24(4):563-572.

[41] Misso NLA, Peacock CD, Watkins DN, Thompson PJ. Nitrite generation and antioxidant effects during neutrophil apoptosis. Free Radic Biol Med. 2000;28(6):934-943. 


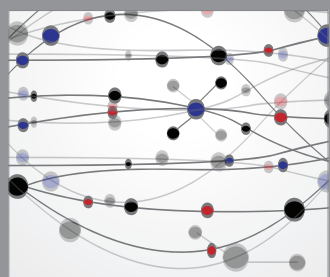

The Scientific World Journal
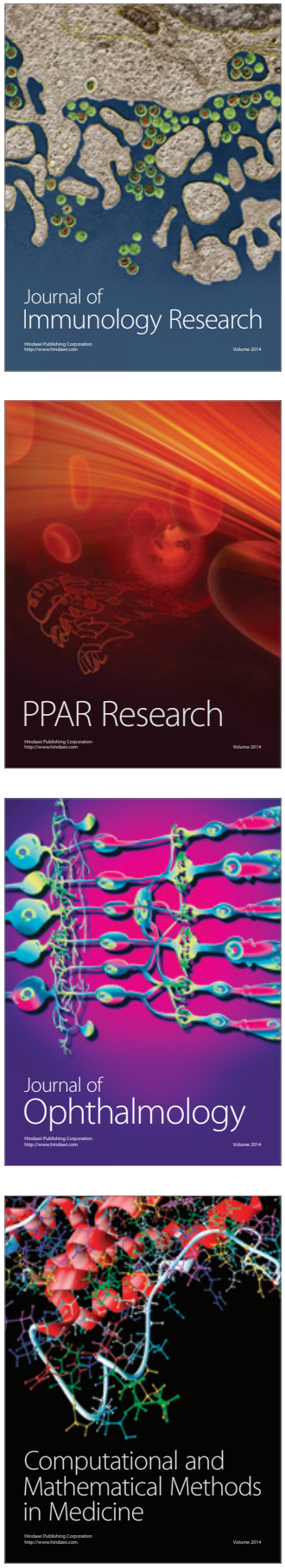



Gastroenterology

Research and Practice
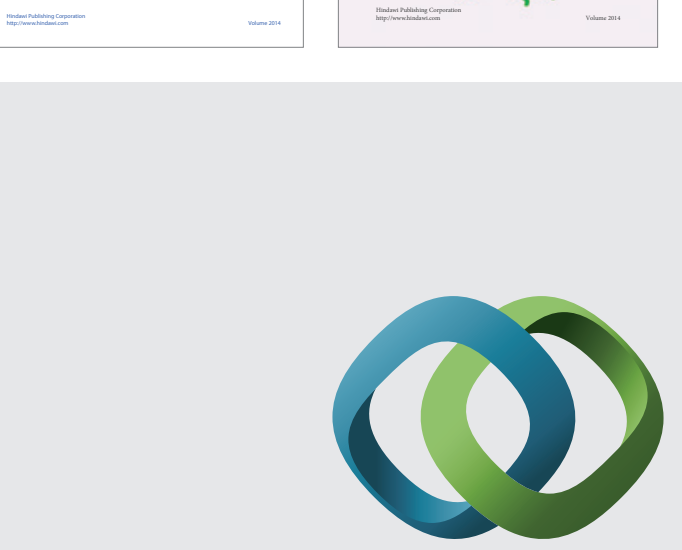

\section{Hindawi}

Submit your manuscripts at

http://www.hindawi.com




Journal of
Diabetes Research

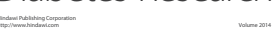

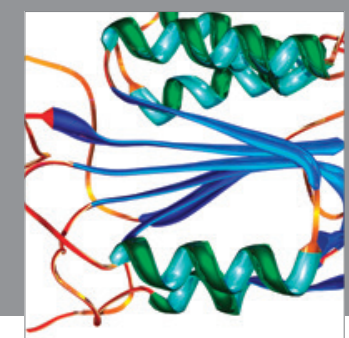

Disease Markers
